\title{
Report on Three Cases of Advance Ovarian Cancer Upon Bangladeshi Population: Successful Management with Bevacizumab Based Chemotherapy
}

\author{
*ME Hoque ${ }^{1}$, S Karim $^{2}$, MMR Siddiqui ${ }^{3}$, T Ahmed ${ }^{4}$
}

\begin{abstract}
Ovarian cancer is an alarming health problem in Bangladesh. The annual mortality rate per 100,000 people from ovarian cancer in Bangladesh has increased by $40.3 \%$ since 1990, an average of $1.8 \%$ a year. Globcan predicts a change in the reported incidence of ovarian cancer from 2912 in year 2012 to 3132 in 2015. Recurrent high-grade ovarian cancer is usually associated with short term survival. There are few guidelines to surgically and medically treat long term survivors with ovarian cancer. We are reporting three cases on advance ovarian cancer patients; all are married, age ranging from 40-60 years, primarily treated with chemotherapy. After that, they were experienced with FDA approved (Nov 14, 2014) monoclonal antibody Bevacizumab (AVASTIN), additionally with chemotherapy.
\end{abstract}

Key Words: Advance ovarian cancer, Chemotherapy, Bevacizumab, Recurrent.

\section{Introduction}

Ovarian cancer is a cancer that forms in an ovary ${ }^{3}$. It results in abnormal cells that have the ability to invade or spread to other parts of the body ${ }^{4}$. When this process begins, there may be no or only vague symptoms. Symptoms become more noticeable as the cancer progresses ${ }^{5,6}$. These symptoms may include bloating, pelvic pain, abdominal swelling, and loss of appetite, among others ${ }^{5}$. Common areas to which the cancer may spread include the lining of the abdomen, lining of the bowel and bladder, lymph nodes, lung, and liver ${ }^{7,8}$. The risk of ovarian cancer increases in women who have ovulated more over their lifetime. This includes those who have never had children, those who begin ovulation at a younger age or reach menopause at an older age ${ }^{9}$. Other risk factors include hormone therapy after menopause, fertility medication, and obesity ${ }^{3}$. About $10 \%$ of cases are related to inherited genetic risk; women with mutations in the genes BRCA1 or BRCA2 have about a $50 \%$ chance of developing the disease. The most common type of ovarian cancer, comprising more than $95 \%$ of cases, is ovarian carcinoma ${ }^{9}$. Screening is not recommended in women who are at average risk, as evidence does not support a reduction in death and the high rate of false positive tests may lead to unneeded surgery, which is accompanied by its own risks ${ }^{10}$. Those at very high risk may have their ovaries removed as a preventive measure ${ }^{5}$. If caught and treated in an early stage, ovarian cancer may be curable. Treatment usually includes some combination of surgery, Targeted therapy, radiation therapy, and chemotherapy 5 . Outcomes depend on the extent of the disease and the subtype of the cancer present ${ }^{9}$. The overall fiveyear survival rate in the United States is $45 \% 11$. Outcomes are worse in the developing world 9 .

1*Prof. Dr. Md. Ehteshamul Hoque, Professor \& Head of Radiation Oncology, Anwer Khan Modern Medical College Hospital

${ }^{2}$ Dr. Shanaz Karim, Assistant Professor of Transfusion Medicine, Dhaka Medical College Hospital, Dhaka

${ }^{3}$ Dr. Md. Mahmudur Rahman Siddiqui, Associate Professor of Medicine, Anwer Khan Modern Medical College Hospital

${ }^{4}$ Dr. Tanvir Ahmed, Medical Officer of Oncology, Anwer Khan Modern Medical College Hospital

*Corresponding Author

Date of submission: 03.02.2017, Date of acceptance: 20.04 .2017

AKMMC J 2017; 8(2) : 157-161 
However, trials of the antibody and VEGF inhibitor bevacizumab, which can slow the growth of new blood vessels in the cancer, have shown promising results ${ }^{12}$. Bevacizumab has been particularly effective in preliminary studies on stage-III and -IV cancer, Bevacizumab can also be combined with platinum chemotherapy, a combination that has had positive preliminary results in PFS, but equivocal results regarding overall survival ${ }^{12}$.

\section{Case 1}

Mrs. X, 62 years muslim lady, normotensive, nondiabetic, non-asthmatic patient hailing from Dhanmondi, Dhaka. In December 2007, the patient had come with one week history of abdominal distension which was progressive in nature. She was investigated and revealed a raised CA125:950, abdominal tap was positive for malignant cells. U/S abdomen revealed right pleural effusion and moderate ascites.

Patient underwent U/S ovary in SGH: uterus was not enlarged. No uterine masses seen. The ovaries were ill defined. Collection of echos seen in right adnexum. This may represent a loop of intestine although an ovarian lesion cannot be excluded. It measures approximately $33 \mathrm{~mm} \times 27 \mathrm{~mm}$ in size. Fluid was present in abdominal and pelvic cavities and in the lung bases. OGD and colonoscopy done for her were normal. Patient underwent ultrasound guided ascetic fluid aspiration, the fluid was then sent for further investigations. Concentration of CA125 was raised to 991. Histology of peritoneal fluid: metastatic adenocarcinoma. Immuno profile: The tumor cells were CK7 positive, calretinin negative, CD1125 focally positive, CDX2 negative. The above findings were in favor of metastatic ovarian carcinoma or peritoneal disease.

Patient underwent abdominal tap and a total of 2.5 liters was drained. Patient was admitted electively for chemotherapy. Planned for 3 cycles of taxol+carboplatin before planning for primary debulking surgery.

After that, from $1^{\text {st }}$ to $6^{\text {th }}$ cycle she got Taxol+Carboplatin followed by THBSO and omentectomy, appendicetomy (histopathologically complete remission). Subsequently disease was progressed in 2009 and she received Carboplatin + Gemzar + Avastin (Bevacizumab) at her $13^{\text {th }}$ cycle of chemotherapy. From $7^{\text {th }}$ to $12^{\text {th }}$ cycle she got Carboplatin + Doxorubicin with acceptable toxicity profile. She was noted to have noted right hypochondrial pain in Aug 2012 with CT PET: Disease recurrence at liver. She thus came to seek review. She had performed an MRI liver which revealed mainly perihepatic and peritoneal disease, no liver parenchymal disease seen. Option of palliative chemotherapy was discussed with patient and her family.

In 2011, she had taken total 6 cycle of chemotherapy $\left(13^{\text {th }}\right.$ to $\left.18^{\text {th }}\right)$. She received Carboplatin + Avastin + Taxol/Gemzar/Taxotare in different stages of her chemotherapy cycle from $19^{\text {th }}$ to $24^{\text {th }}$ in 2012. In 2013, She had been started her $25^{\text {th }}$ cycle with only Avastin ( $500 \mathrm{mg}$ ). She received Neulastim (1amp) additionally with chemotherapy in her $28^{\text {th }}$ cycle, because of neutropenia. She has taken her last chemotherapy on Jan 09, 2017. It was her $47^{\text {th }}$ cycle. She is continuing her treatment, Though she is a patient of aggressive Advance Ovarian Cancer (stage 4), her disease progression is successfully managed by her current treatment protocol.

\section{Case 2}

Ms. Tahera, 37 years old, regularly menstruating, got admitted to UHL under Obs \& Gynae dept on 28.06.2009 with the complaints of swelling of a abdomen for 2 months and vague abdominal discomfort, bloating and weight loss for 6 months. She is normotensive, nondiabetic. $\mathrm{P} / \mathrm{A} / \mathrm{E}$ reveals a large solid mass about $16 \times 18 \mathrm{~cm}$ occupying umbilical, hypogastic \& left \& right lumber region. Investigations revealed $\mathrm{Hb}: 9.3 \mathrm{gm} / \mathrm{dl}, \mathrm{CA}-125$ : 207.88 U/ml, betah CG: 102.71 Miu/ml. CT Scan showed bilateral ovarian neoplasm. Probably malignant with abdominal lymphadenopathy bilateral hydronephrosis and hydroureter. Her total hysterectomy with bilateral salphingo-opherectomy with infracolic omentectomy were done on 30.06.2009. Histopathology showed clear cell Adenocarcinoma of ovary. Both peritoneal fluid and omentum were also positive for malignancy. The patient was transferred to oncology dept. for further 
treatment on 12.07.2009. She was given 6 cycle of chemotherapy of carboplatin $350 \mathrm{mg}$ DI, paclitaxel $240 \mathrm{mg}$ DI and six cycles of Targeted therapy Bevacizumab (Avastin) $400 \mathrm{mg}$ dose treatment and it was non-event full. In the meantime her $\mathrm{CBC}$, LFT, RFT CEA, CA125 was normal. The patients were then evaluated by PET-CT scan in Singapore and FBG positive pelvic node was identified. Patients was then given option for second look surgery but patients party refused surgery and agreed to Receive Further chemotherapy and targeted therapy. Then she was given 3 cycle of second line, Avastin, Gemcitabine \& Oxaloplatin. Review MRI was done and showed no change in abdominal (Lymphoid) lesion. But CA-125 is in good control and patients are Asymptomatic. Latest CA-125 result is $2.56 \mathrm{U} / \mathrm{ml}$ (19.02.2014). In 2016(18.03.2016) whole body FDG PET-CT scan was done. Now patients come follow-up 6 monthly $\&$ patients iaAsymptomatic.

\section{Case 3}

Patients Age 45. Post menopausal, nondiabetic HTN presented with sudden respiratory dissension, abdominal discomfort and ascites. She was investigated and revealed a raised CA-125.

CT. Scan shows lung metastasis and mass in right ovary. Emergency $\mathrm{CT}$ guided aspiration was done. Exploratory laparotomy \& total abdominal hysterectomy with bilateral salpingo-ophorectomy were done. Histopathology showed cysts adenocarcinoma with positive ascetic fluid for malignant cell. Her CA-125 was 5000. She was diagnosed as a stage 4 ovarian cancer.

Then she received adjuvant chemotherapy 6 cycles with the targeted therapy bevacizumab (Avastin). Her CA-125 cancer marker returned to normal. After 12 months later her CA-125 level rises and then again received 6 cycle of paclitaxel, gemcitibine, cisplatin and targeted therapy bevacizumab (Avastin).

She received 6 cycle adjuvant chemotherapy like carboplatin plus paclitaxel and targeted therapy Bevacizumab (Avastin). Every three months she comes to the chamber for follow-up and patients is asymptomatic.

\section{Discussion}

Globally, as of 2010 , about 160,000 people died from ovarian cancer, up from 113,000 in $1990^{14}$. As of 2014, more than 220,000 diagnoses of epithelial ovarian cancer were made yearly ${ }^{15}$. Ovarian cancer is most commonly diagnosed after menopause ${ }^{15}$, between the ages of 60 and $64.90 \%$ of ovarian cancer occurs in women over the age of 45 and $80 \%$ in women over $50^{16}$. The annual mortality rate per 100,000 people from ovarian cancer in Bangladesh has increased by $40.3 \%$ since 1990, an average of $1.8 \%$ a year. Though this has been the trend overall, adjust the filters at the top of the visualization to see how the mortality rate for ovarian cancer has changed over time for women of specific age groups in Bangladesh ${ }^{17}$.

Cancer is one of the leading causes of morbidity and mortality in Bangladesh. The estimated cancer load is 1.2 million with an incidence of 2000000 , a prevelance of 800000 and mortality at 150000 . Cancer incidence is expected to double in the next 20 years $^{18}$. In a survey of 117 cancer patients in Dhaka, $8.1 \%$ had ovarian cancer ${ }^{19}$.

We report the case of women with advanced and high-grade epithelial ovarian cancer who survived for 10 years despite recurrences. They received complex and highly sophisticated oncologic care in Bangladesh. While the mean five-year overall survival from advanced epithelial ovarian cancer is $20 \%$. There is a subset of women who are longterm survivors.

In a review of 251 women with advanced stage ovarian cancer univariate analysis revealed that FIGO stage 3 and stage 4 , elevated CA125, and suboptimal de bulking were significant in reducing duration of progression free survival(PFS) and overall survival (OS).

One of the challenges for cancer patients in Bangladesh is the tremendous cost of care. Women with ovarian cancer consume a large proportion of healthcare resources ${ }^{20}$. Bangladesh has developed a pluralistic healthcare system, which includes government services, multiple NGOs and robust private sector ${ }^{21}$. Majority of Bangladeshi citizens cannot afford healthcare and do not have access to 
complex care our patients received ${ }^{22}$. Development of universal healthcare insurance must be part of the strategy in Bangladesh for complex care such as ovarian cancer ${ }^{23,20}$.

Prognosis is not good in advance stage of ovarian cancer. Treatment options in recurrence metastasis stage is limited for ovarian carcinoma. So conventional treatment regimen show poor response and survival. So newer molecule need to investigate that enhance the efficacy ovarian carcinoma in advance stage ${ }^{24}$.

Vascular endothelial growth factor (VEGF) and angiogenesis are important promoter of ovarian cancer progression ${ }^{25,26}$. Both co-relate directly with the extent of disease and inversely with progression free survival ${ }^{27,28}$ and overall survival ${ }^{29,30,31}$ often in dependently of known prognostic factors ${ }^{32,33}$.

Bevacizumab, a humanize VEGF-neutralizing monoclonal antibody, inhibits tumor angiogenesis ${ }^{4}$. We investigate the integration of bevacizumab with our conventional treatment protocol.

In the first case- The patients was 62 yrs. old and first she diagnosed ovarian cancer at 2007. Sequentially she taken conventional chemotherapy and targeted therapy bevacizumab. At 10 years later she comes to dr. chamber regularly and she continued the single agent bevacizumab.

In case $2 \& 3$ another two patients they also diagnosed stage 4 ovarian cancer. They also get all the standard treatment and they come to the chamber 3 months intervals

In our finding from three case studies, bevacizumab is one of the important monoclonal antibody showed single agent activity in woman with recurrent ovarian cancer.

Conflict of interest: We have no conflict of interest.

\section{References}

1. Global Health Data Exchange (ghdx. healthdata. org), NHS Choices(nhs. uk), and The World Bank(data.worldbank.org).

2. Globocan 2015 Ovarian cancer in Bangladesh accessed June 20, 2015.

3. "Ovarian Cancer Prevention (PDQ $\left.{ }^{\circledR}\right) "$. NCI. December 6, 2013. Retrieved 1 July 2014.

4. "Chronic pelvic pain" (PDF). ACOG. Retrieved 11

5. "Ovarian Epithelial Cancer Treatment (PDQ $\left.{ }^{\circledR}\right) "$. NCI. 2014-05-12. Retrieved 1 July 2014.

6. Ebell, MH; Culp, MB; Radke, TJ. A Systematic Review of Symptoms for the Diagnosis of Ovarian Cancer. American journal of preventive medicine. March 2016;50 (3): 38494. doi:10.1016/j.amepre.2015.09.023. PMID 26541098.

7. "Treating advanced cancerresearchuk.org.. Retrieved 2015-05-16ovarian cancer". www

8. Ruddon, Raymond W. (2007). Cancer biology (4th ed.). Oxford: Oxford University Press. p. 223. ISBN 9780195175431

9. World Cancer Report 2014. World Health Organization. 2014. Chapter 5.12. ISBN 9283204298 .

10. Moyer VA. Screening for ovarian cancer: U.S. Preventive Services Task Force reaffirmation recommendation statement. Annals of Internal Medicine. Dec 2012; 157 (12): 900-4. doi:10.7326/0003-4819-157-11-20121204000539. PMID 22964825.

11. "SEER Stat Fact Sheets: Ovary Cancer". NCI. Retrieved 18 June 2014.

12. Jayson GC, Kohn EC, Kitchener HC, et al. Ovarian cancer. Lancet. October 2014; 384 (9951): 1376-88. doi: 10.1016/S01406736(13)62146-7. PMID 24767708.

13. Lozano R, Naghavi M, Foreman K, et al. Global and regional mortality from 235 causes of death for 20 age g Jayson GC, Kohn EC, Kitchener HC, Ledermann JA (October 2014). "Ovarian cancer". Lancet. Dec 2012; 384 (9951): 1376-88. doi:10. 1016/S0140-6736(13)62146-7. PMID 24767708. 
14. A systematic analysis for the Global Burden of Disease Study 2010". Lancet.2010; 380 (9859): 2095-128. doi:10.1016/S0140-6736(12)617280. PMID 23245604

15. Jayson GC, Kohn EC, Kitchener HC, et al. Ovarian cancer. Lancet. October 2014; 384 (9951): 1376-88. doi:10.1016/S0140-6736(13) 62146-7. PMID 24767708.

16. "Ovarian cancer". DynaMed. June 18, 2015.

17. Global-disease-burden.healthgrove.com/1/ 37072/Ovarian-Cancer-in-Bangladesh.

18. National cancer control strategy and plan of action 2009-2015. Accessed June 21, 2015.

19. Hasan AHMN, Uddin Md M, Rafiquzzaman $\mathrm{Md}$, et al. Distributation types of cancer and pattern of cancer treatment among the patients of various hospitals in Dhaka division; Bangladesh Intl Res J Pharma, 2012; 3: 219-222.

20. Kim K, Hernlund E, Hernadi Z, et. Al. Treatment pattern, healthcare utilization, and cost of ovarian cancer in central and Eastern Europe using a Delphi panel based on a retrospective chart review. Int $\mathrm{J}$ Gynecol cancer. 2013; 23: 823-832.

21. Chowdhory AM, Bhuiyan A, Chowdhory ME, et al. Bangladesh: Innovation for universal Health Coverage: The Bangladesh Paradox: Exceptional health achievement despite economic poverty. Lancet 2013; 382: 1734-1745.

22. Hussain SM. Comprehensive update on cancer Scenario of Bangladesh. South Asian J Cancer, 2013; 2: 279-284.

23. DuXL, Sun CC, Milan MR, et al. Ethnic differences in socioeconomic Status, diagnosis, treatment and survival among older women with epithelial ovarian cancer. Int J Gynecol Oncol. 2013: 24: 342-351.

24. Shahana P, Farzana I, Tracilyn H, et al. The managemen Of Ovarian cancer In Bangladesh: Austin J Obstet Gynecol 2015; 2(4): 1047.
25. Xu L, Yoneda J. Herrera C, Wood J, et al. Inhibition of Malignant Ascities and growth of human ovarian carcinoma by oral administration of a potent inhibitor of the vascular endothelial growth factor tyrosine kinase. Int Oncol 2000; 16: 445-54.

26. Belotti D, Calcagno C, Garofalo A, et al. Vaslular endothelial growth factor stimulates organ specific host matrix me-tallopproteinase expression and ovarian cancer invasion. Mol Cancer Res 2008; 6: 525-34.

27. Robert A, Burger M.D., Mark $F$ et al. Incorporation of Bevacizumab in The Primary Treatmant of Ovarian Cancer. The New England Journal of Medicine 365; 26: 24732482.

28. Hollings worth HC, Kohn EC, Stein-Berg SM, et al. Tumour Angiogenesis in Advance stage ovarian carcinoma. AM J Pathol 1995; 147: 33-41.

29. Gasparini G, viale $\mathrm{G}$, Bonoldie $\mathrm{E}$ et al. Prognostic and predictive value of tumor angiogenesis in ovarian carcinomas. Int $\mathbf{J}$ Cancer 1996; 69: 205-11.

30. Shen GH, Ghazizadeh m, Kawanami O, et al. prognostic significance of vascular endothelial growth factor expression in human ovarian carcinoma. Br J cancer 2000; 83: 196-203.

31. Duncan TJ, Al-Ahar A, Rolland P, et al. Vascular endothelial growth factor expression in ovarian cancer: A model for targated use of novel therapies. Clin Cancer Res 2008; 14: 3030-5.

32. Siegel R, ward E, Brawley O, et al. Cancer Statistics, 2011: The impact of eliminating Socioeconomic and racial dis-parities on premature cancer death. CA-cancer J Clin 2011; 61: 212-36. 